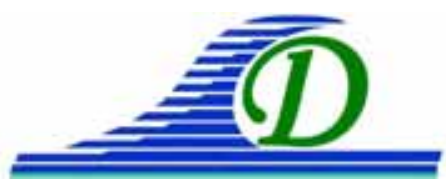
XIII ${ }^{\text {èmes }}$ Journées Nationales Génie Côtier - Génie Civil
Dunkerque, 2-4 juillet 2014

DOI:10.5150/jngcgc.2014.061 ～(C) Editions Paralia CFL

disponible en ligne - http://www.paralia.fr - available online

\title{
Profiler 3.1 XL, un logiciel gratuit pour la construction et l’analyse de profils topographiques dans Microsoft Excel(C)
}

\section{Olivier COHEN ${ }^{1}$}

1. Université de la Nouvelle-Calédonie, Centre des Nouvelles Études sur le Pacifique (EA 4242), 145 avenue James Cook, BP R4, 98851 Nouméa Cedex, Nouvelle-Calédonie.

olivier.cohen@univ-nc.nc

\section{Résumé :}

Profiler 3.1 XL est la dernière version d'un logiciel gratuit de construction et d'analyse descriptive de profils topographiques, notamment de profils de plage ; il est cependant utilisable pour d'autres types de relief. C'est une extension de Microsoft Excel@ $\mathbb{C}$. La programmation est réalisée en Visual Basic pour Applications. Ce programme a été conçu pour être manipulé facilement par un public non-spécialiste de la topométrie et de la géométrie, au sein de la communauté scientifique, des étudiants et des gestionnaires s’intéressant à la mesure et l'évolution du littoral. Il propose de nombreuses fonctions : importation et mise en forme de fichiers en provenance d'appareils de mesure, préparation des données, création de graphiques, analyses descriptives simples, analyses plus perfectionnées (notamment identification et description d'ondulations -bermes, sandwaves- description détaillée multicritères, comparaison de profils deux à deux). Un mode d'emploi détaillé est fourni avec le programme.

Mots-clés : Microsoft Excel@, Logiciel, Profil topographique, Ergonomie, Représentation graphique, Calcul de paramètres descriptifs, Analyse morphologique, Comparaison.

\section{Introduction}

Les profils de plage sont, aujourd'hui, très utilisés dans les études de description et d'évolution des rivages. Ce sont des représentations pratiques de la morphologie du rivage: ces coupes topographiques à grande échelle permettent, par exemple, de distinguer dune, plage, barres intertidales, voire subtidales si le profil se prolonge sur l'avant-côte (KRAUS, 2005). Dans les analyses diachroniques, on peut y suivre les mouvements transversaux du trait de côte, cet objet aux multiples définitions qui peut être difficile à identifier sur des photographies aériennes (BOAK \& TURNER, 2005) ; on peut également y mesurer les variations altitudinales du niveau de la plage, en déduire l'évolution des volumes pour calculer des bilans sédimentaires. Enfin, ces profils peuvent donner lieu à des modélisations visant à comprendre l'évolution de la plage sous l'effet des conditions hydrodynamiques et aérodynamiques ou à en prédire l'évolution dans le temps (KEIJESERS et al., 2014). 


\section{Thème 3 - Instrumentation, mesures, imagerie et télédétection}

Les profils peuvent être mesurés dans le cadre d'études scientifiques et universitaires à court terme. Ils le sont de plus en plus souvent, dans le cadre d'observatoires du littoral et/ou du trait de côte à plus long terme (JEANSON et al., 2010 ; SUANEZ et al., 2012) qui accumulent, au bout de quelques années une grande quantité de données. La base de données JARKUS du Rijkswaterstaat néerlandais contient ainsi des profils levés plusieurs fois par an, tous les $500 \mathrm{~m}$, depuis 1965. Il est donc nécessaire de disposer d'outils informatiques pour leur analyse.

Les données peuvent être collectées in situ avec des instruments très variés : parfois très simples (cadres pour mesurer des distances et des dénivellations, EMERY, 1961 ; PULEO et al., 2008), un peu plus techniques (niveaux de chantier, théodolites) ou de haute technologie (tachéomètres, DGPS). Certaines données sont extraites de Modèles Numériques de Terrain calculés par exemple avec des mesures LiDAR (COHEN \& GARDEL, 2011 ; KLEMAS, 2011). Tous ces levés nécessitent différents traitements géométriques pour pouvoir obtenir des informations sur des longueurs, des volumes, des évolutions, etc. (BRABANT et al., 2012).

Profiler 3.1 XL est la dernière version d'un logiciel gratuit et francophone pour la construction et l'analyse descriptive de profils topographiques, notamment de profils de plage, mais il est utilisable pour d'autres types de relief. C'est une extension de Microsoft Excel@ à l'instar du célèbre logiciel d'analyse de données et de statistique XLSTAT (site web). La programmation est réalisée en Visual Basic pour Applications. Cette "boite à outils" a été conçue pour être manipulée facilement par un public non-spécialiste de la topométrie et de la géométrie, au sein de la communauté scientifique, des étudiants et des gestionnaires s'intéressant à la mesure et l'évolution du littoral. Ce logiciel est couramment utilisé dans des travaux d'étudiants et d'enseignants-chercheurs de différentes universités (ex. : UNC, ULCO, UAG). Profiler 3.1 XL est téléchargeable à l'adresse suivante : https://www.dropbox.com/sh/h8exwy52cdmx8zd/V5rXC3Gzn0.

D'autres logiciels de construction et analyse de profils topographiques existent. "The Beach" (CHANDRASEKAR et al., 2009) est un programme en Visual Basic. Il permet de traiter simplement des mesures de distances et d'altitude issues de la méthode d'EMERY (1961). Il est bien adapté pour les équipes ne disposant que peu de moyens techniques et financiers. D’autres solutions sont plus perfectionnées et intégrées à un système d'archivage, de mise à disposition et de représentations graphique et cartographique des données, par exemple le projet MADDOG en Bretagne (SUANEZ et al., 2012). D’autres programmes sont plus évolués encore. Cependant certains sont anciens et n’ont pas été mis

à jour depuis longtemps et ne sont plus compatibles avec les dernières versions du système d'exploitation Windows, comme c'est le cas pour le programme BMAP (site web), Beach Morphology Analysis Package du corps des ingénieurs de l'armée américaine, (SOMMERFELD et al., 1993). Enfin, d'autres sont plus complexes d'utilisation et/ou coûteux : par exemple, BPAT, Beach Profile Analysis Toolbox, développé au sein du 


\section{XIII ${ }^{\text {èmes }}$ Journées Nationales Génie Côtier - Génie Civil \\ Dunkerque, 2-4 juillet 2014}

NIWA, National Institute of Water and Atmospheric Research en Nouvelle-Zélande (site web) ; modules d'AutoCAD comme COVADIS (site web).

\section{Principales fonctions et ergonomie}

Profiler 3.1 XL propose des fonctions que l'on peut classer en plusieurs catégories :

- importation et mise en forme de fichiers en provenance de certains modèles d'appareils de mesure,

- préparation de données (ex. : conversion de coordonnées XYZ en distances et Z),

- création de graphiques (répartition des points en plan, profil),

- analyses simples (ex. : calcul de gisement, de pente, de volume, interpolation d'altitude le long du profil, découpage du profil en sections d'égale longueur pour les analyses plus poussées),

- analyses plus perfectionnées (ex. : identification et description d'ondulations -bermes, barres, sandwaves- description détaillée multicritère, comparaison de profils deux à deux).

Après le démarrage, un menu déroulant "PROFILER 3.1 XL" apparait dans l’onglet "Compléments" (figure 1). Comme pour celles de Excel, pour activer une fonction, il faut d'abord sélectionner une plage de données, peu importe son emplacement sur la feuille de calcul active, puis cliquer sur la fonction dans le menu. Quand une boite de dialogue est affichée et que la fonction est validée, celle-là reste à l'écran afin que l'on n’ait pas besoin de la sélectionner de nouveau dans le menu si on veut la réutiliser. On peut donc afficher plusieurs boites de dialogue en même temps. Cela est pratique pour les traitements "à la chaine". Pour les fonctions simples, les résultats sont donnés dans une boite de dialogue. Pour les fonctions plus élaborées, ils sont affichés dans une nouvelle feuille de calcul. La feuille de calcul de l'utilisateur n'est pas modifiée, sauf pour les fonctions graphiques.

Travailler dans Microsoft Excel $@$ présente plusieurs avantages : c’est un programme très répandu, facile d'utilisation, proposant des fonctions que notre logiciel va exploiter. Il est possible par exemple d'importer les données à traiter et d'enregistrer les résultats dans de nombreux formats (texte, tableurs, bases de données) : en exportant les coordonnées XY des points d'un profil en format .csv on peut les spatialiser dans un logiciel de SIG. La création et la personnalisation des diagrammes y est également très aisée : on peut ainsi indiquer des marges d'erreur de mesure verticale en spécifiant des valeurs de barres d'erreur pour les points d'un profil (très agrandies pour être visibles sur la figure 1).

Il est possible de personnaliser certaines opérations via la fonction "Options". Par exemple celles de l'onglet "Graphiques 1" permettent de paramétrer les titres et noms des axes des graphiques de types "Répartition des points en plan" et "Profils" pour les créations ultérieures. L’option "Calcul du coefficient de détermination" $\left(\mathrm{r}^{2}\right)$ est utile pour quantifier la qualité de l'alignement des points d'un profil le long d'un axe : ce coefficient varie entre 0 et 1 ; quand il tend vers 1 , les points sont bien alignés, quand il 
s'en éloigne et que les points sont répartis de façon sinueuse autour de l'axe, cela peut traduire un problème de mise à l'horizontale d'un théodolite ou d'un tachéomètre.

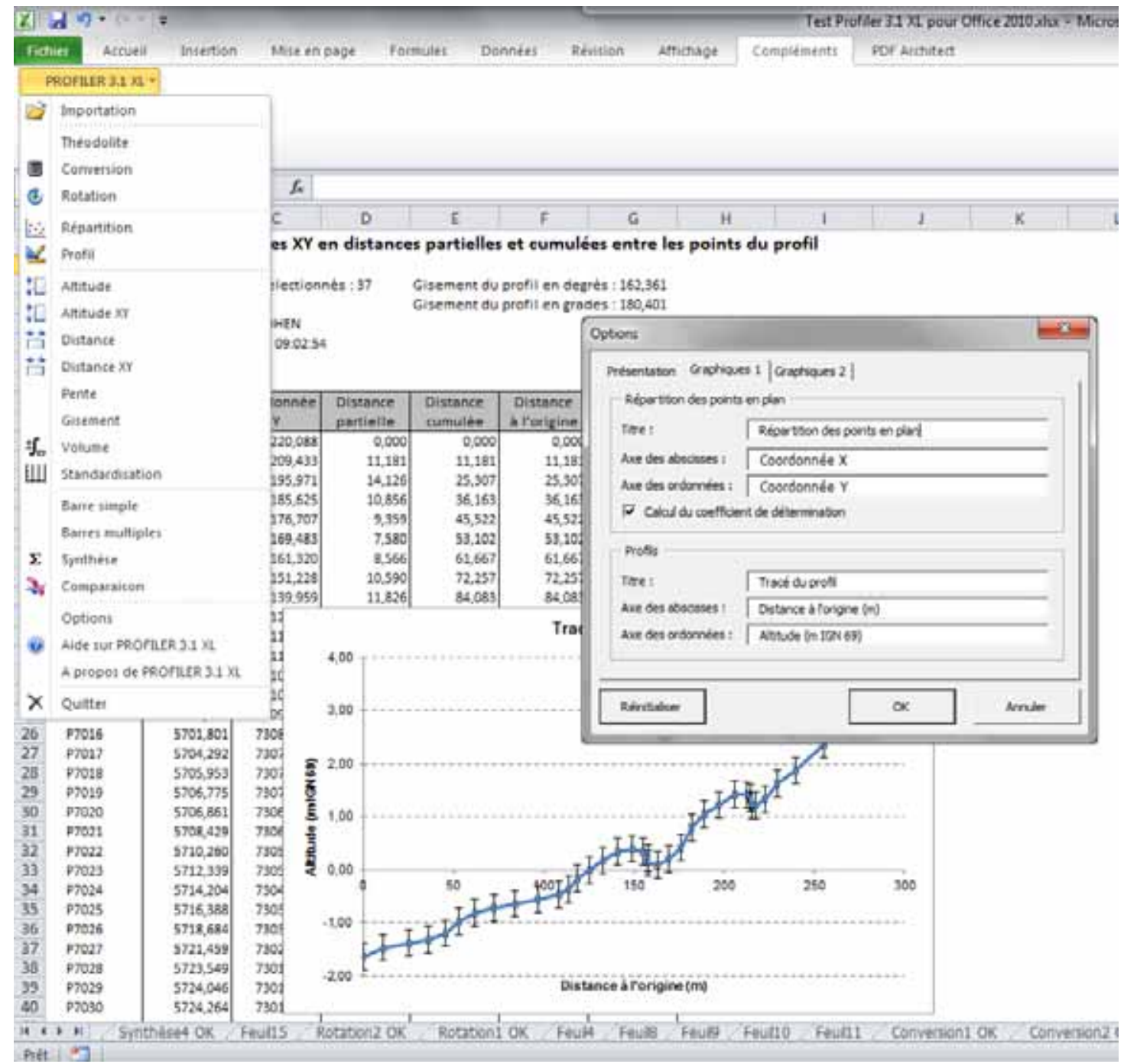

Figure 1 : le menu des fonctions de Profiler 3.1 XL

Exemples de tracé d'un profil topographique et de la boite de dialogue des options.

Profiler 3.1 XL comporte des routines pour détecter les erreurs dans les données sélectionnées (ex. : si la plage de données n'a pas le nombre de colonnes nécessaire au calcul ou si elle contient des cellules vides ou de texte) et les erreurs de saisie dans les paramètres des boites de dialogues (par exemple si on a saisi une valeur hors du champ de celles de la variable à traiter). Un message précise à chaque fois le type d'erreur commise.

Le logiciel propose une aide en ligne avec sommaire, mots-clés indexés et fonction de recherche. Un mode d'emploi de 40 pages est également fourni en format pdf. Il contient tous les détails sur l'installation et l'utilisation du logiciel. 


\section{XIII ${ }^{\text {èmes }}$ Journées Nationales Génie Côtier - Génie Civil \\ Dunkerque, 2-4 juillet 2014}

\section{Exemple de la fonction "Barres multiples"}

Cette fonction permet de détecter automatiquement et décrire les ondulations positives sur toute la longueur ou une section d'un profil topographique (ex. : barres inter- ou subtidales, bancs, rides). Elle se fonde sur les écrits de BRANDER (1999) et MASSELINK \& ANTHONY (2001). Ces ondulations sont définies par rapport à la pente de la plage qui peut être modélisée de plusieurs façons (figure 2) :

- par régression linéaire simple des altitudes en fonction des distances qui modélise une pente homogène, l'équation de la régression est $y^{\prime}=a x+b$ où le coefficient directeur $a$ représente la pente ; ce choix est adaptée pour les plages lisses,

- par régression polynomiale qui permet de calculer une pente non homogène avec des inflexions (une pour un polynôme de rang 2, deux pour un polynôme de rang 3, etc.) ; ce choix est adapté pour des plages convexes ou concaves.

Deux graphiques sont affichés sur la feuille de résultats (figure 2) : l'un présente le profil topographique classique, la pente/régression de la zone d'étude et son équation, l'autre fait apparaître les barres avec une composante verticale exprimée en hauteur (résidu par rapport à la régression/pente moyenne).

Les résidus (différence entre les altitudes réelles et théoriques le long de la régression) sont calculés : on assimile ceux positifs aux barres (ridges) et ceux négatifs aux creux (runnels). La limite entre une barre et une bâche est donc le croisement entre le profil mesuré et la régression (figure 3 ). On peut donc calculer la position de ces points et déterminer l'extension horizontale des barres. La crête de barre correspond au résidu positif maximal, sa hauteur est la différence entre l'altitude sur le profil et celle sur la régression. Deux types de "volume" de barre sont calculés (figure 3) : l'un correspond à la surface située entre le profil et la régression, l'autre à celle entre le profil et une altitude-plancher définie par l'utilisateur.

\section{Exemple de la fonction "Comparaison"}

Cette fonction permet de comparer deux profils topographiques. Pour être comparables, les profils sont tout d'abord découpés à la même longueur et rééchantillonés en sections égales. La feuille de résultats (figure 4) contient :

- en en-tête, la valeur du pas de rééchantillonage (découpage) et le plancher de référence, les longueurs individuelles et la longueur commune, les "volumes" et la différence de "volume" des deux profils.

- dans le tableau de gauche, les données de départ,

- dans le tableau de droite, les distances standardisées, les coordonnées Z théoriques des deux profils et leur différence (P2 - P1), les volumes des différentes sections des deux profils et leur différence,

- deux graphiques sont affichés: l'un présente la superposition des deux profils (courbes) et leur différence (barres verticales), l'autre fait apparaître uniquement la différence entre les deux profils (c’est un zoom sur le diagramme précédent). 
Thème 3 - Instrumentation, mesures, imagerie et télédétection

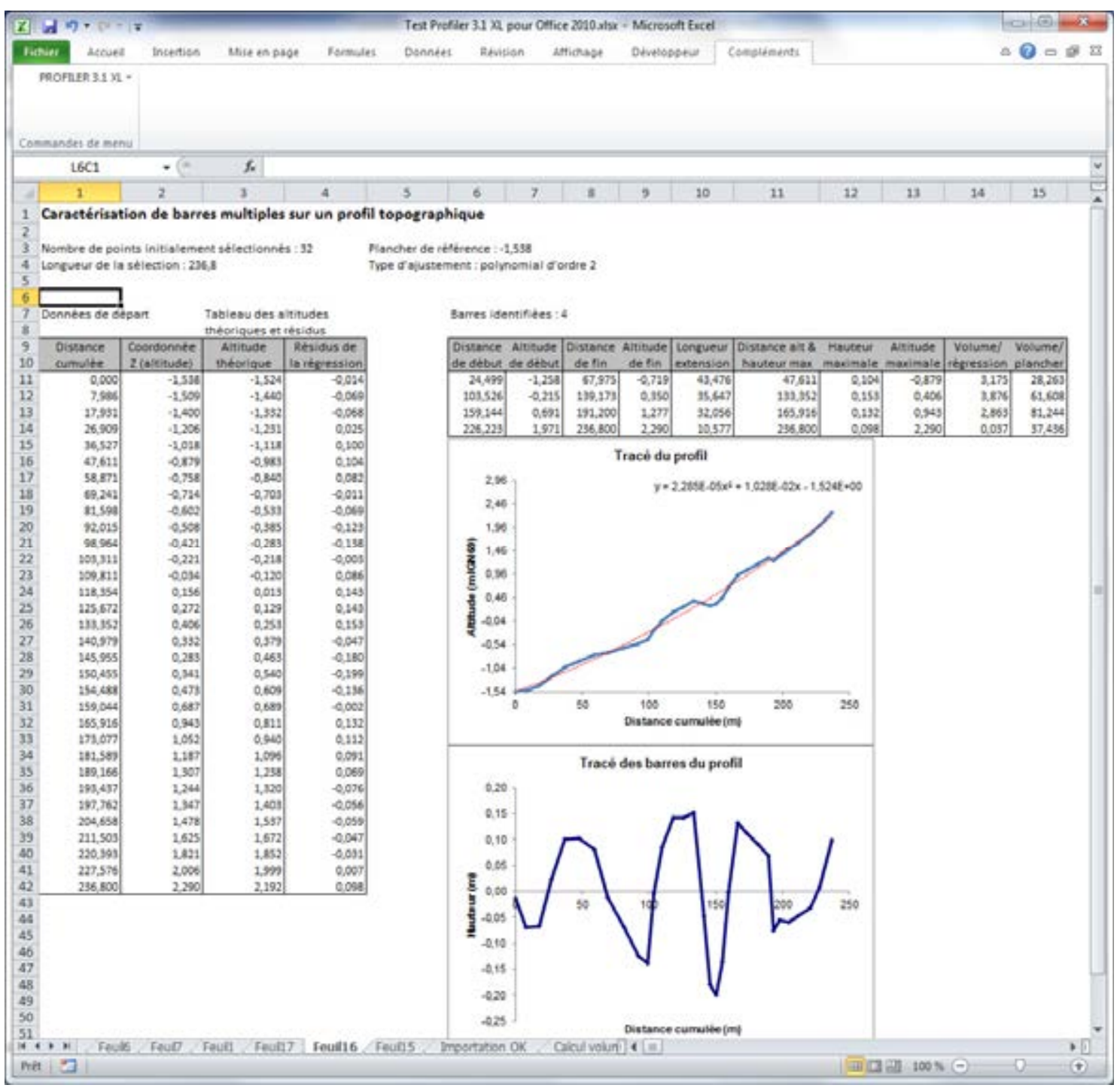

Figure 2. Exemple de la boite de dialogue et de la feuille de résultats de la fonction "Barres multiples".
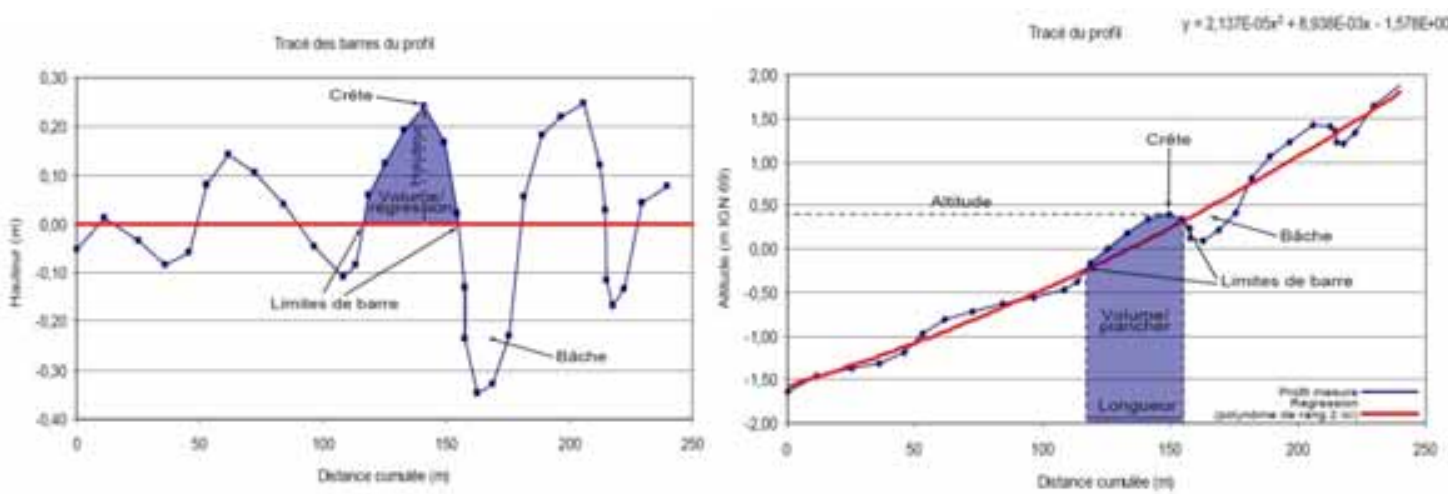

Figure 3. Principes de calcul des limites, crête et du volume des barres. 


\section{XIII ${ }^{\text {èmes }}$ Journées Nationales Génie Côtier - Génie Civil \\ Dunkerque, 2-4 juillet 2014}

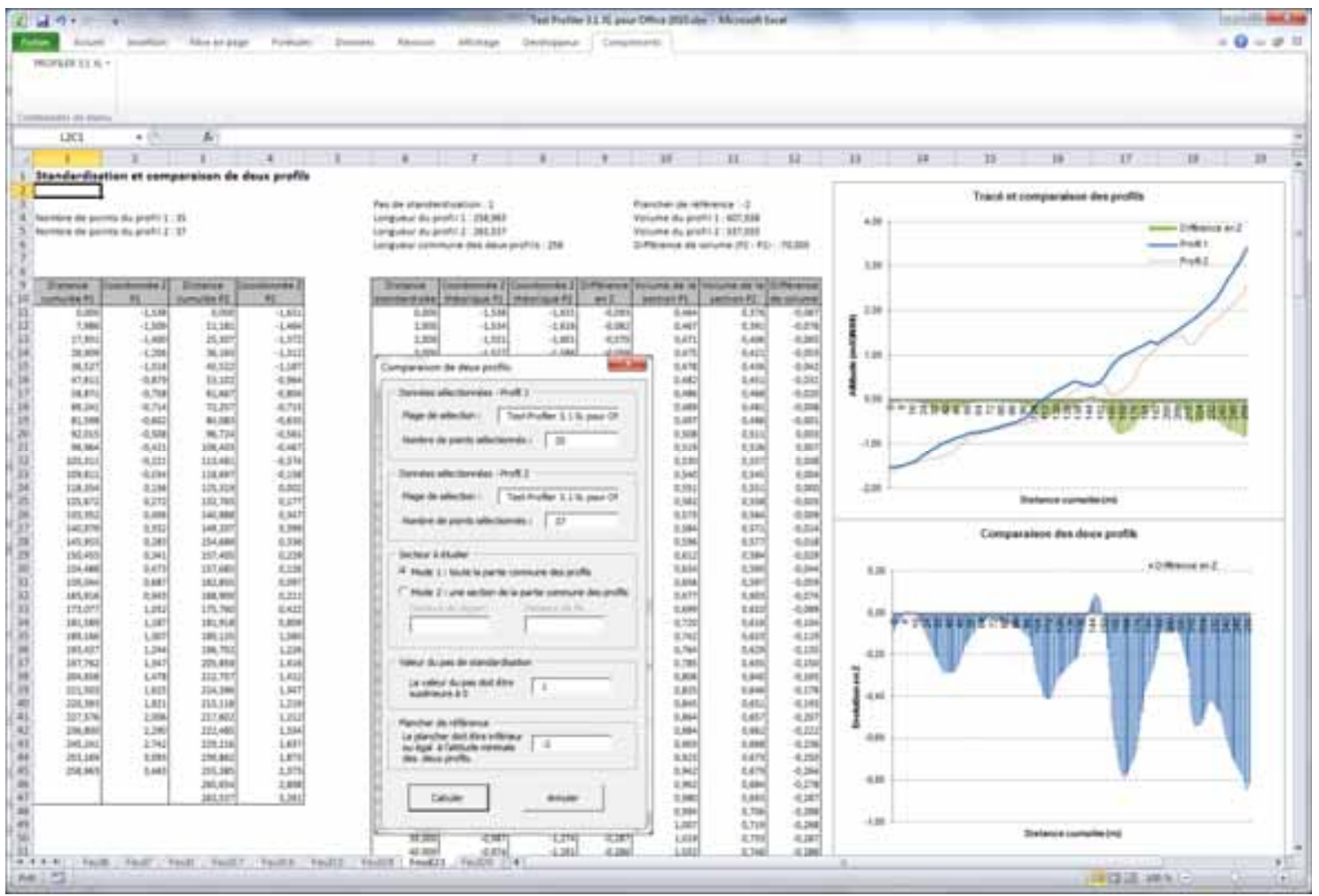

Figure 4. Exemple de la boite de dialogue et de la feuille de résultats de la fonction

"Comparaison".

\section{Conclusion et perspectives}

Profiler 3.1 XL est un logiciel gratuit, très simple et rapide d'utilisation. Nous projetons d'ores et déjà de développer de nouvelles fonctions, notamment de vérification de l'alignement, de comparaison et de synthèse statistique de plusieurs profils. Mais surtout, nous envisageons une suite de développement plus collaborative et une adaptation au tableur de la suite bureautique LibreOffice pour permettre une diffusion encore plus large et totalement gratuite du logiciel.

\section{Références}

BMAP (site web). http://chl.erdc.usace.army.mil/chl.aspx?p=s\&a=SOFTWARE;32

BOAK E.H., TURNER I.L. (2005). Shoreline definition and detection: a review. Journal of Coastal Research, Vol. 21(4), pp 688-703. http://dx.doi.org/10.2112/03-0071.1

BPAT (site web). http://www.niwa.co.nz/our-science/coasts/tools-and-resources/tides/bpat BRABANT M., PATIZEL B., PIEGLE A., MÜLLER H. (2012). Topographie opérationnelle. Eyrolles, Paris, 396 p.

BRANDER R.W. (1999). Field observations on the morphodynamic evolution of a lowenergy rip-current system. Marine Geology, Vol. 157, pp 199-217. http://dx.doi.org/10.1016/S0025-3227(98)00152-2 
Thème 3 - Instrumentation, mesures, imagerie et télédétection

CHANDRASEKAR N., SHEIK MUJABAR P., RAJAMANICKAM G.V. (2009). A simple computer program for the analysis of beach morphology and morphodynamics. International Journal of Geoinformatics, Vol. 5(4), pp 63-67.

COHEN O., GARDEL A. (2011). De l'analyse des cartes anciennes à l'altimétrie laser aéroportée : l'évolution des techniques de suivi du trait de côte. Exemples sur la Côte d'Opale. In actes du colloque Observation des écosystèmes marin et terrestre de la Côte d’Opale : du naturalisme à l'écologie, Union des Océanographes Français, Paris, pp 153-169.

COVADIS (site web). http://www.geo-media.com/covadis.htm

EMERY K.O. (1961) A simple method of measuring beach profiles. Limnology and Oceanography, ${ }^{\circ}$ 6, pp 90-93. Disponible en ligne sur http://www.aslo.org/lo/toc/vol_6/issue_1/0090.pdf JEANSON M., DOLIQUE F., ANTHONY E.J. (2010). Un réseau de surveillance des littoraux face au changement climatique en milieu insulaire tropical : l'exemple de Mayotte. VertigO, Vol. 10(3). http://dx.doi.org/10.4000/vertigo.10512

KEIJSERS J.G.S., POORTINGA A., RIKSEN M.J.P.M., MAROULIS J. (2014) Spatiotemporal variability in accretion and erosion of coastal foredunes in the Netherlands: regional climate and local Topography. PLoS ONE, Vol. 9(3), e91115, pp 1-11. http://dx.doi.org/10.1371/journal.pone.0091115

KLEMAS V. (2011). Beach profiling and LIDAR bathymetry: an overview with case studies. Journal of Coastal Research, 27, pp 1019-1028. DOI: 10.2112/JCOASTRES-D-11-00017.1 KRAUS N.C. (2005). Beach profile. In SCHWARZ M.L. (ed) Encyclopedia of Coastal Science, Springer, pp 169-172. http://dx.doi.org/10.1007/1-4020-3880-1_37

MASSELINK G., ANTHONY E.J. (2001). Location and height of intertidal bars on macrotidal ridges and runnels beaches. Earth Surface Processes and landforms, Vol. 26, pp 759-774. http://dx.doi.org/10.1002/esp.220

PULEO J.A., PEARRE N.S., HE L., SCHMIED L., O'NEAL M., PIETRO L.S., FOWLER M. (2008). A single-user subaerial beach profiler. Journal of Coastal Research, 24, n4, pp 1080-1086. http://dx.doi.org/10.2112/06-0665.1

SOMMERFELD B. G., MASON J. M., LARSON M. and KRAUS N. C. (1993). Beach Morphology Analysis Package (BMAP). Beach Nourishment Engineering and Management Considerations, American Society of Civil Engineers, pp 162-175.

XLSTAT (site web). http://www.xlstat.com/fr/

SUANEZ S., GARCIN M., BULTEAU T., ROUAN M., LAGADEC M., DAVID L. (2012) Les observatoires du trait de côte en France métropolitaine et dans les DOM. EchoGéo Vol. 19. http://dx.doi.org/10.4000/echogeo.12942 